\title{
METHOD TO DETECT SIMILARITY IN SOURCE CODE IN PROGRAMS USED IN ENGINEERING
}

\author{
SIR-ALEXCI SUAREZ ${ }^{1} \&$ ALBERT MIYER SUAREZ ${ }^{2}$ \\ ${ }^{1}$ Engineering Faculty, University Francisco of Paula Santander Ocaña, Colombia \\ ${ }^{2}$ Engineering Faculty. University of Pamplona, Colombia
}

\begin{abstract}
In this research, a method is presented to find similarity between source codes of the Pseint language, which is used in the teaching of programming in mechanical, civil and systems engineering. The code is quantized with values from 0 to 255 forming histograms for each tokens extracted. Pearson's correlation is used to find similarity throughout the code, in the use of variables and control structures. The correlation value greater than 0.8 determines the similarity between the different programs. This method can help the tutor to find similarities, but it can also reorient the teaching by analyzing the reason why the similarity is happening between different developers of the software.
\end{abstract}

KEYWORDS: Source code, Pseint language, similarity software, token quantification

Received: Oct 06, 2020; Accepted: Oct 26, 2020; Published: Dec 03, 2020; Paper Id.: IJMPERDOCT202084

\section{INTRODUCTION}

Software coding is not an area just for computer scientists or exclusively for courses or careers with an emphasis on systems. Therefore, today it is being implemented in the majority of higher level academic programs and educational institutions at the basic and intermediate levels, through the teaching of programming. Some universities use programming as a practical training in Engineering, carrying out mechanical simulations using blocks with the aim of solving a problem in the mechanical engineering career (Monjelat \& Rodríguez, 2018). Biology is included in the curriculum due to advances in biotechnology and computer science where it is essential to use it for a greater contextualization of the subject (Carvajal-Rodríguez, 2015). And if all academic programs are analyzed where statistical calculations have to be made, they are found with programs such as $\mathrm{R}$, which allows an orderly administration of the data and the delivery of results where it offers the possibility of visualizing them graphically (Siddiqui, 2018), positioning it at the forefront in this type of statistical software; In addition, as it is a free software, it gives the possibility of being immediately incorporated into companies, research centers and educational institutions. If we start from the previous base, it can be said that it is not only necessary to take a course to handle software, it is also convenient to understand how we code our instructions. The teaching of coding could be measured at the end in a quantitative way, knowing the performance of the students through their final grade; however, it is appropriate to use techniques that allow reviewing, guiding and finding similarities between the source codes of the programs in order to take appropriate corrections that improve the quality of teaching.

When talking about similarity between source codes, it can be understood that plagiarism and intellectual fraud may exist (Beethoven, 2012), with all the consequences that can occur in losses of millions of dollars and at an 
educational level with academic sanctions. However, what is intended is to find the similarities in the works and tests carried out in order to incorporate processes that allow the student to improve their logic in solving exercises that need to take it to an appropriate coding according to the programming language. Techniques for detecting plagiarism use computer packages that allow quantifying the percentage of plagiarism using mathematical counting formulas and spatial position of the text content. Within these you can name Copyleaks which allows you to select the search according to whether it is educational or business; if it is a school or university, in addition to allowing a review in several languages and, its plagiarism descriptors are: identical, similar text or number of bibliographic sources (Copyleaks, 2020). The Plagium software performs the process by analyzing the number of identical or similar texts according to a number of questions that students must answer (Septet Systems, 2020). The Turnitin software uses the number of words regardless of their length to determine the level of similarity (Moreno, 2018).Text plagiarism software is not functional when it comes to analyzing the coding of the programs because their similarity is based on finding identical words and in the coding, two words can be written in a totally different way, however they are identical due to the declaration or instruction in the program. Within the models used in source codes are the statistic and the n-gram (Bensalem et al., 2014; Stamatatos, 2009); the first calculates the similarity from a vector of characteristics and the second uses a bag of words which it stores in a vector to compare the tokens of a source program.

The objective of this article is to find the similarity between source codes when using the Pseint software, using the histogram as a characteristic descriptor, in such a way that each word or character extracted is converted into a tokens with a gray level value, where different Tokens can be converted into a single one using a quantization value between 0 and 255 as if it were a digital grayscale image, to later apply a correlation based on the quantization of the tokens.

\section{METHODOLOGY}

To use the histogram as a measure descriptor, the text it contains must be extracted from each source code and converted to grayscale, giving a value according to the reserved words of the language where they can be found: functions, operators, data types, conditional and repetitive structures; in addition to the variables or expressions created by the programmer. When mentioning a histogram, we are talking about an image, in such a way that the values of the image can be quantified, in this case the characters or tokens are converted into pixels for each of the gray values, "the histogram of an image It consists of the number of pixels that the image has for each of the gray levels. It is represented by a Cartesian coordinate axis (Alegre et al., 2003, p. 33). In the Figure 1 the histogram of an image can be observed, and the gray values from 0 to 255 to quantify each value of the existing code in an image, therefore 256 values are enough to represent the coding of a program in the teaching cycle by means of Pseint, and is the amount of colors that the human eye can perceive in a certain way. The programs to be analyzed are encoded with Pseint (2020) a pseudo-language in Spanish which is used in most Latin American countries due to its simplicity and the teaching resources it contains. And the scripts are made in Matlab because it is a very powerful language for handling matrices and vectors, which are used to form the histogram and statistical operations. It is known that the histogram is not useful when recognizing two different images because they can have the same histogram, however in the technique that is exposed from source code (PSC) to grayscale it works as an appropriate descriptor to recognize the similarity of encoding, which allows to interpret the distribution of the PSC instructions. 


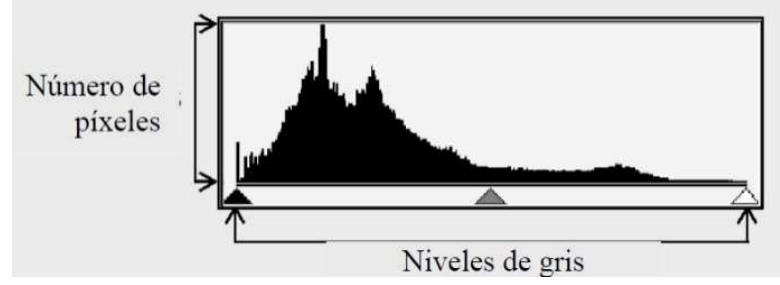

Figure 1: Histogram representation of an image (Alegre et al., 2003, p. 33)

The sample consisted of 73 programs. And in the figure 2, you can see the process that is carried out to compare the PSCs. Each of these programs varies in length and amount of instructions, as well as the location which try to hide the similarity.

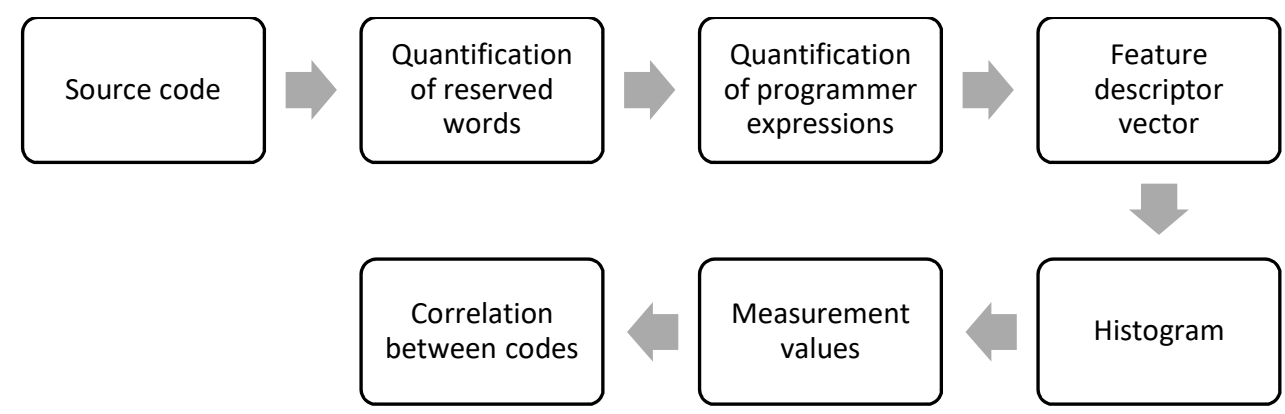

Figure 2: Stages of extraction of measurement values

\subsection{PSC quantification}

The PSC is analyzed and reserved words are extracted which include the three previous types, in addition the variables given in the user expressions are also quantified. At Table 1 , they show the values they take in a range from 0 to 255 , where the reserved words always have a fixed value and the programmer's words are created as the code is analyzed.

Table 1: Quantification of reserved words

\begin{tabular}{|c|c|c|c|c|c|c|c|c|c|}
\hline 0 & 1 & 2 & 3 & 4 & 5 & 6 & 7 & 8 & 9 \\
\hline Algoritmo & finalgoritmo & definir & como & real & entero & carácter & lógico & escribir & leer \\
\hline 10 & 11 & 12 & 13 & 14 & 15 & 16 & 17 & 18 & 19 \\
\hline si & sino & finsi & según & hacer & de otro modo & para & mientras & finmientras & repetir \\
\hline 20 & 21 & 22 & 23 & 24 & 25 & 26 & 27 & 28 & 29 \\
\hline hasta que & para & hasta & con paso & finpara & finsegun & funcion & finfuncion & Mod & entonces \\
\hline 30 & 31 & 32 & 33 & 34 & 35 & 36 & 37 & 38 & 39 \\
\hline O & NO & abs & trunc & redonc & rc & pi & exp & sen & cos \\
\hline
\end{tabular}

The quantification process is carried out by extracting each of the words through a lexical analysis and then converting them to tokens and then quantifying it with a gray value, forming a vector for each PSC. From the previous code, the variables are quantified with the following values: Product with 42, quantity with 43, price with 44, discount with 45 and total discount with 46 . Subsequently, for each line, a table is created with the values that appear in the code according to the identation of the program; for this program there is an indentation of 3 , therefore 3 columns are extracted; the indentation is 
given by the number of structures used in a nested way, plus the first indentation after the start of the program. Then the amount of each equal value in the quantification is added and placed in its respective row according to the value of the corresponding gray level for each one (see table 2).

Table 2: Program 1 quantification

\begin{tabular}{|c|c|c|c|c|c|c|c|c|}
\hline Line & C1 & $\mathrm{C} 2$ & $\mathrm{C3}$ & Histogram & Quantity C1 & Quantity C2 & Quantity C3 & Final value \\
\hline 1 & 0 & & & 0 & 1 & 0 & 0 & 1 \\
\hline 2 & 8 & & & 1 & 1 & 0 & 0 & 1 \\
\hline 3 & 2 & 3 & 5 & 2 & 2 & 0 & 0 & 2 \\
\hline 4 & 2 & 3 & 4 & 3 & 0 & 2 & 0 & 2 \\
\hline 5 & 8 & & & 4 & 0 & 0 & 1 & 1 \\
\hline 6 & 8 & & & 5 & 0 & 0 & 1 & 1 \\
\hline 7 & 8 & & & 6 & 0 & 0 & 0 & 0 \\
\hline 8 & 8 & & & 7 & 0 & 0 & 0 & 0 \\
\hline 9 & 8 & & & 8 & 19 & 0 & 0 & 19 \\
\hline 10 & 9 & 42 & & 9 & 4 & 0 & 0 & 4 \\
\hline 11 & 13 & 42 & 14 & 10 & 0 & 0 & 0 & 0 \\
\hline 12 & & & & 11 & 0 & 0 & 0 & 0 \\
\hline 13 & 8 & & & 12 & 0 & 0 & 0 & 0 \\
\hline 14 & 8 & & & 13 & 1 & 0 & 0 & 1 \\
\hline 15 & 9 & 43 & & 14 & 0 & 0 & 1 & 1 \\
\hline 16 & 44 & & & 15 & 1 & 0 & 0 & 1 \\
\hline 17 & 45 & 44 & & 16 & 0 & 0 & 0 & 0 \\
\hline 18 & 46 & 43 & 46 & 17 & 0 & 0 & 0 & 0 \\
\hline 19 & 8 & & & 18 & 0 & 0 & 0 & 0 \\
\hline 20 & 8 & & & 19 & 0 & 0 & 0 & 0 \\
\hline 21 & & & & 20 & 0 & 0 & 0 & 0 \\
\hline 22 & 8 & & & 21 & 0 & 0 & 0 & 0 \\
\hline 23 & 8 & & & 22 & 0 & 0 & 0 & 0 \\
\hline 24 & 9 & 43 & & 23 & 0 & 0 & 0 & 0 \\
\hline 25 & 44 & & & 24 & 0 & 0 & 0 & 0 \\
\hline 26 & 45 & 44 & & 25 & 1 & 0 & 0 & 1 \\
\hline 27 & 46 & 43 & 45 & 26 & 0 & 0 & 0 & 0 \\
\hline 28 & 8 & & & 27 & 0 & 0 & 0 & 0 \\
\hline 29 & 8 & 46 & & 28 & 0 & 0 & 0 & 0 \\
\hline 30 & & & & 29 & 0 & 0 & 0 & 0 \\
\hline 31 & 8 & & & 30 & 0 & 0 & 0 & 0 \\
\hline 32 & 8 & & & 31 & 0 & 0 & 0 & 0 \\
\hline 33 & 9 & 43 & & 32 & 0 & 0 & 0 & 0 \\
\hline 34 & 44 & & & 33 & 0 & 0 & 0 & 0 \\
\hline 35 & 45 & 44 & & 34 & 0 & 0 & 0 & 0 \\
\hline 36 & 46 & 43 & 45 & 35 & 0 & 0 & 0 & 0 \\
\hline 37 & 8 & & & 36 & 0 & 0 & 0 & 0 \\
\hline 38 & 8 & 46 & & 37 & 0 & 0 & 0 & 0 \\
\hline 39 & 15 & & & 38 & 0 & 0 & 0 & 0 \\
\hline 40 & 8 & & & 39 & 0 & 0 & 0 & 0 \\
\hline
\end{tabular}




\begin{tabular}{|c|c|c|c|c|c|c|c|c|}
\hline 41 & 25 & & & 40 & 0 & 0 & 0 & 0 \\
\hline 42 & 1 & & & 41 & 0 & 0 & 0 & 0 \\
\hline & & & & 42 & 0 & 2 & 0 & 2 \\
\hline & & & & 43 & 0 & 6 & 0 & 6 \\
\hline & & & & 44 & 3 & 3 & 0 & 6 \\
\hline & & & 45 & 3 & 0 & 2 & 5 \\
\hline
\end{tabular}

The program has 42 lines and a quantization of 47 gray level values; therefore it is not necessary to use the 256 values. All programs are quantized in the same way, regardless of the length of each program, because in the end the vector is already delimited to 256 .

\section{RESULTS}

The tests carried out find similarity in the gray levels once the code has been converted through quantification, evaluating three possibilities: complete code, control structures and the variables used. To show the quantification technique, 5 examples of PSC out of 73 are presented, to do the whole process until the correlation data in the similarity.

\subsection{Source code analysis}

Each PSC can give us an idea of the similarity between them, in the table 3 part of the code that can be object of similarity is extracted. The first three programs were delivered with adequate operation according to the problem, programs 3 and 4 were not delivered in their entirety, as some calculations were missing.

Table 3: Program code fragments

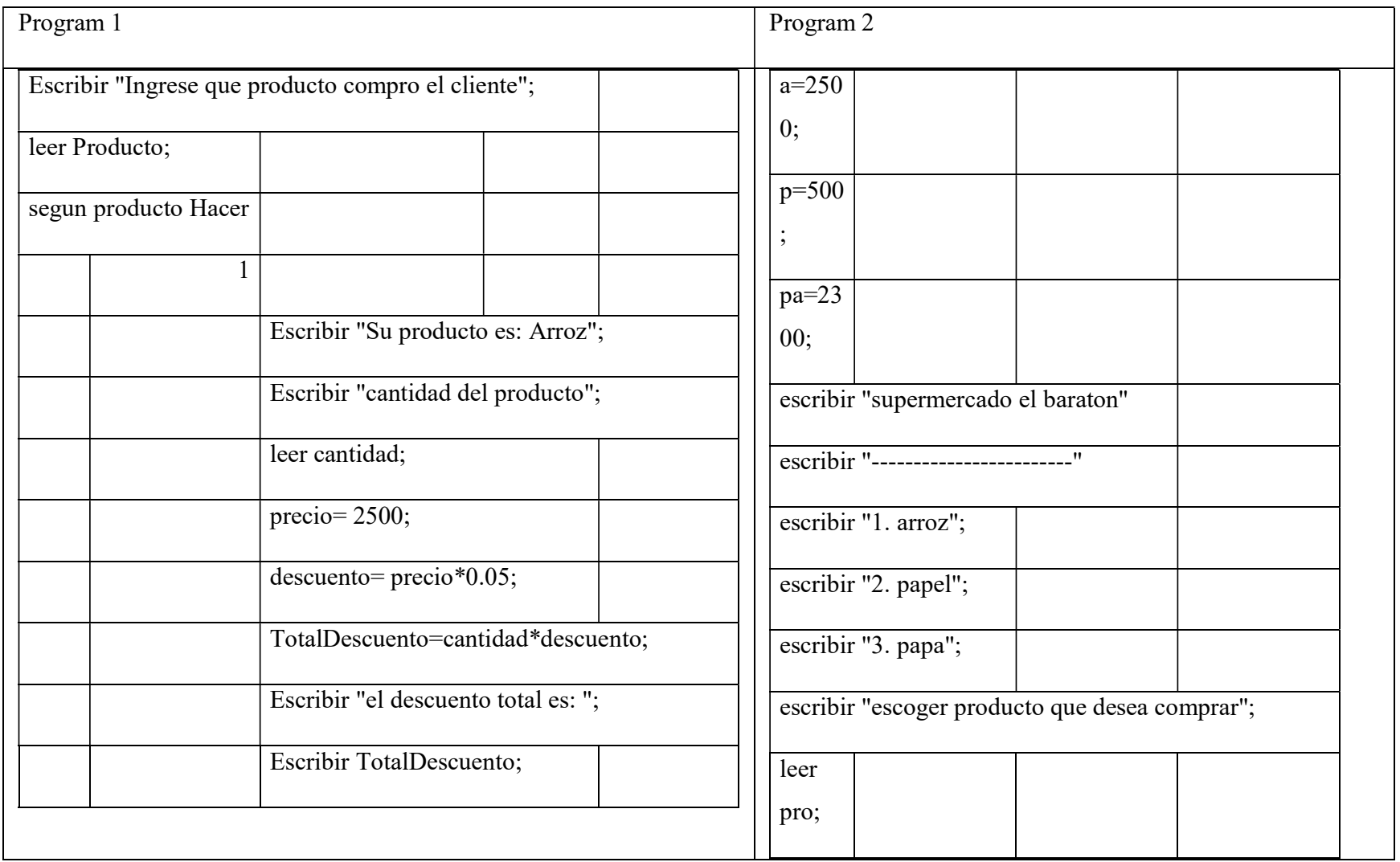




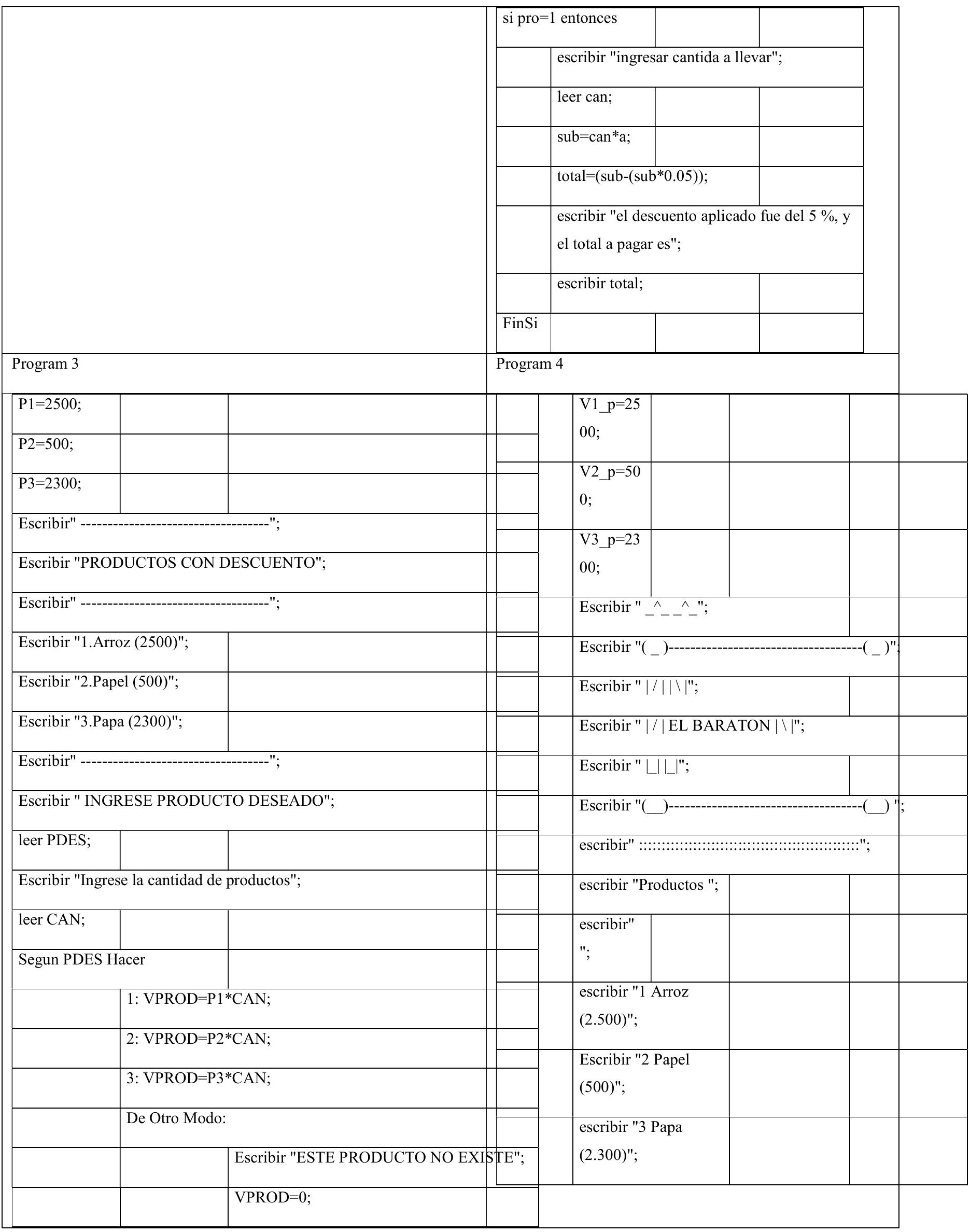




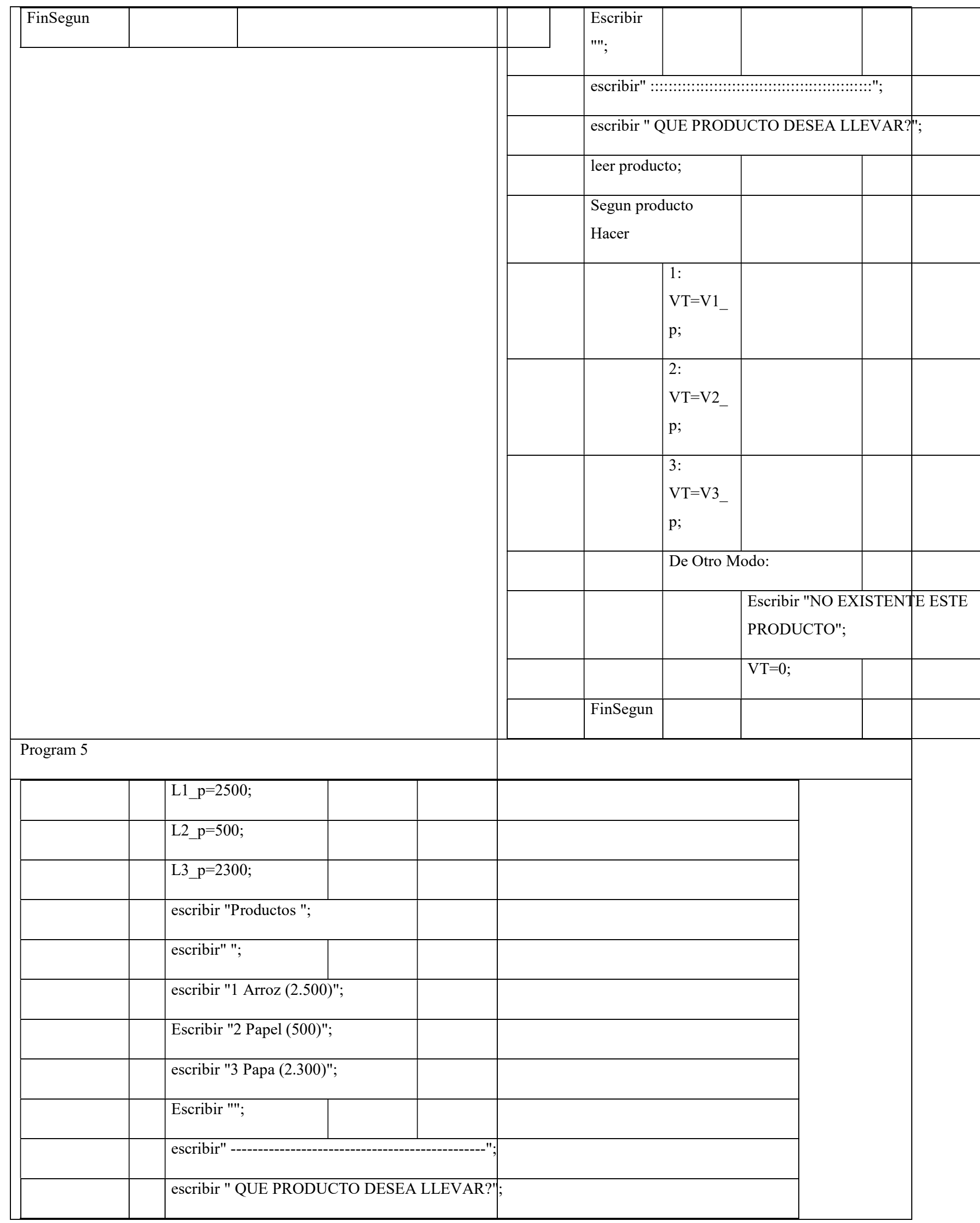




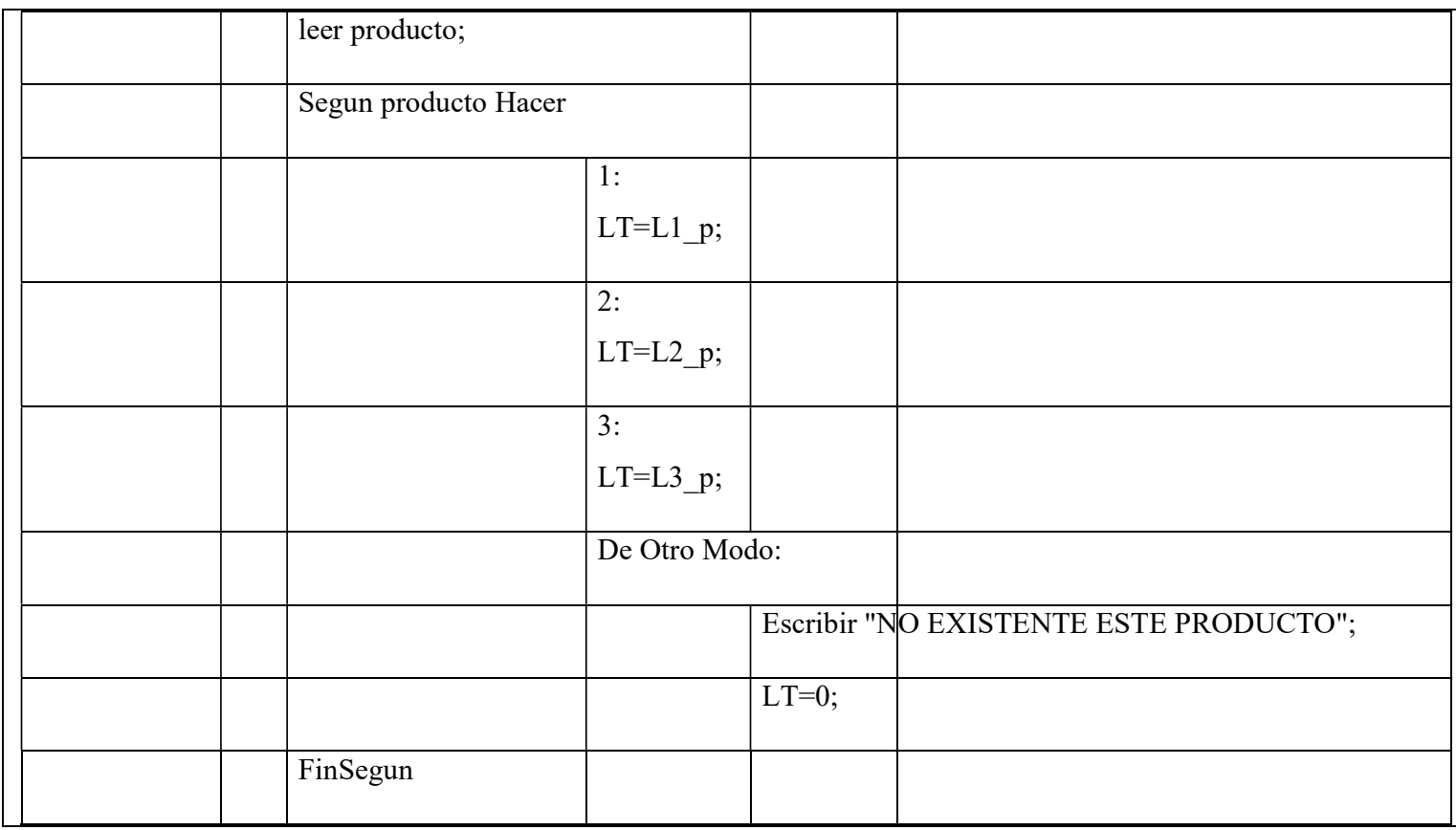

In program 1 and 2 different structures are used, in one menus are created while the other displays and requests products separately; program 1 with 2, 3 and 5 use the same structures but their way and logic of solving the problem is very different; When comparing program 2 with 3,4 and 5, it can be determined that they have no similarity, they only have similarity in the creation of the product menu, something that is not a determining factor; program 3 is very similar to 4 and 5 , with the difference that the program was delivered completely; While 4 and 5 do not solve the problem in its entirety, finally program 4 and 5 are the same, although 4 is longer and it can be seen that they try to hide their similarity by entering new lines with messages on the screen, through the instruction Write. As a result the 3, 4 and 5 are similar; 3 and 4 are the same with the same errors in the lack of completion of the program, although due to the length of the programs, not all the code is presented to indicate the deficiencies of 4 and 5 with respect to 3 . At table 4 the quantification values of the 5 programs are presented; When reviewing each line, it can be detected that from lines 13 to 25 and from lines 42 to 45 a similarity is established in the values of programs 3,4 and 5; Furthermore, on lines 46 to 49, programs 4 and 5 continue to maintain the same value.

Table 4: Final quantification values per program

\begin{tabular}{|r|r|r|r|r|r|}
\hline Histograma & Value P1 & \multicolumn{1}{|c|}{ ValueP2 } & Value P3 & Value P4 & Value P5 \\
\hline 0 & 1 & 1 & 1 & 1 & 1 \\
\hline 1 & 1 & 1 & 1 & 1 & 1 \\
\hline 2 & 2 & 2 & 2 & 2 & 2 \\
\hline 3 & 2 & 2 & 2 & 2 & 1 \\
\hline 4 & 1 & 1 & 1 & 1 & 1 \\
\hline 5 & 1 & 1 & 1 & 1 & 0 \\
\hline 6 & 0 & 0 & 0 & 0 & 0 \\
\hline 7 & 0 & 0 & 0 & 0 & 14 \\
\hline 8 & 19 & 15 & 19 & 22 & 1 \\
\hline 9 & 4 & 4 & 2 & 1 & 0 \\
\hline 12 & 0 & 3 & 0 & 0 & \\
\hline
\end{tabular}




\begin{tabular}{|c|c|c|c|c|c|}
\hline 13 & 1 & 0 & 2 & 2 & 2 \\
\hline 14 & 1 & 0 & 2 & 2 & 2 \\
\hline 15 & 1 & 0 & 2 & 2 & 2 \\
\hline 25 & 1 & 0 & 2 & 2 & 2 \\
\hline 29 & 0 & 3 & 0 & 0 & 0 \\
\hline 32 & 0 & 1 & 0 & 0 & 0 \\
\hline 42 & 2 & 2 & 2 & 3 & 3 \\
\hline 43 & 6 & 2 & 2 & 3 & 3 \\
\hline 44 & 6 & 1 & 2 & 3 & 3 \\
\hline 45 & 5 & 4 & 3 & 3 & 3 \\
\hline 46 & 6 & 6 & 4 & 4 & 4 \\
\hline 47 & 0 & 9 & 8 & 5 & 5 \\
\hline 48 & 0 & 6 & 6 & 0 & 0 \\
\hline 49 & 0 & 0 & 2 & 0 & 0 \\
\hline
\end{tabular}

A matrix is created with the quantization values to be able to apply the image analysis techniques to the histogram for its later automatic analysis.

\subsection{Automatic analysis by quantification}

Three tests are carried out to detect the similarity because some may be more conclusive than others, these tests are based on the correlation that exists between all the values of the histogram points, such as: complete program, variables that go from the line 42 to the end of the code and control structures that go from line 10 to 27 . The value 10 that belongs to the Write instruction is eliminated, as it is not relevant to find a similarity. Pearson's correlation coefficient can be calculated using equation 1.

$$
\rho(A, B)=\frac{1}{N-1} \sum_{i=1}^{N}\left(\overline{\frac{A_{l}-\mu_{A}}{\sigma_{A}}}\right)\left(\overline{\frac{B_{l}-\mu_{B}}{\sigma_{B}}}\right)
$$

Where the mean and standard deviation of each observation are calculated, in this case of each level of gray by program code. The results of the table 5, show that P1 has no similarity with P2, however it shows very high values with P4 and $\mathrm{P} 5$, which is not correct, since they are not equal programs; Likewise, P2 and P3 present a high correlation which is not exact either, while P3, P4 and P5 if they are selected for their high correlation, and P4 and P5 are practically the same with a correlation of 0.9811 , as a conclusion when analyzing the whole program it can present false positives.

Table 5: Full code similarity

\begin{tabular}{|l|l|l|l|l|}
\hline P1 & P2 & Q3 & Q4 & P5 \\
\hline 1 & 0.7553 & 0.8329 & 0.9259 & 0.9158 \\
\hline 0.7553 & 1 & 0.9123 & 0.8268 & 0.8340 \\
\hline 0.8329 & 0.9123 & 1 & 0.9390 & 0.9324 \\
\hline 0.9259 & 0.8268 & 0.9390 & 1 & 0.9811 \\
\hline 0.9158 & 0.8340 & 0.9324 & 0.9811 & 1 \\
\hline
\end{tabular}


In the results of the table 6 , it can be seen that $\mathrm{P} 1$ has no similarity with any of the programs; $\mathrm{P} 2$ and $\mathrm{P} 3$ are quite similar; P3 has no similarity to P4 and P5; P4 and P5 are identical. As a conclusion, although it improves its results, it still presents false positives.

Table 6: Code similarity by variables

\begin{tabular}{|l|l|l|l|l|}
\hline 1 & -0.2536 & -0.5141 & 0.4284 & 0.4284 \\
\hline-0.2536 & 1 & 0.9335 & 0.4557 & 0.4557 \\
\hline-0.5141 & 0.9335 & 1 & 0.2454 & 0.2454 \\
\hline 0.4284 & 0.4557 & 0.2454 & 1 & 1 \\
\hline 0.4284 & 0.4557 & 0.2454 & 1 & 1 \\
\hline
\end{tabular}

In the results of the table 7 , it is observed that $\mathrm{P} 1$ and $\mathrm{P} 2$ do not have similarity with any program; $\mathrm{P} 3$ has a high similarity with P4 and P5, even with the same similarity value because they are the same programs and P4 and P5 are identical. In conclusion, when performing a correlation with control structures, the process is effective to detect the similarity between codes. And it can be used to detect similarity at the same time regardless of the number of PSCs in place, reducing review time by a classroom tutor.

Table 7: Code similarity by control structures

\begin{tabular}{|l|l|l|l|l|}
\hline 1 & 0.5286 & 0.7460 & 0.5509 & 0.5509 \\
\hline 0.5286 & 1 & 0.1063 & -0.0698 & \\
\hline 0.7460 & 0.1063 & 1 & 0.9668 & 0.9668 \\
\hline 0.5509 & -0.0698 & 0.9668 & 1 & 1 \\
\hline 0.5509 & -0.0698 & 0.9668 & 1 & 1 \\
\hline
\end{tabular}

It must be remembered that the correlation is expressed by the similarity in its order and not by the quantification of the absolute values. So when you do the tests visually checking the PSC values and they had the same value in their gray levels it could be thought that they are the same; as it happened with the tests of the variables where their values were equal but the results did not offer similarity. To determine similarity, a threshold must be declared; In this case, 0.8 has been selected due to the results obtained in the 73 CSPs where the existence of similar programs was already known, and they yielded a value above this threshold. When using this technique, it does not matter if the identification name of the variables has been changed or their length is extended or decreased, even if their location changes in the number of lines, since it is ordered by their gray level value of the histogram. Of the 73 programs analyzed, 11 were found to be similar, which were already known in advance, therefore the quantification method in gray levels offers acceptable results and the calculation time is extremely fast.

\section{CONCLUSIONS}

With the method of quantification of the source code in gray level values, similarity of the programs delivered in the classroom can be detected, as Pseint is an almost exclusive programming language for teaching. The correlation that determines the greatest similarity is when the control structures used in the code are analyzed. This method can help the tutor to find similarities, but it can also reorient the teaching by analyzing the reason why the similarity is happening between different developers of the software. The correlation threshold is 0.8 determined by the number of codes where similarity was found. In a future work, the logical operators should be added, which are not included in the correlation process and can improve the detection together with the control structures. 


\section{REFERENCES}

1. Alegre, E., Sánchez, L., Fernandez-Diaz, R., \& Mostaza, J. (2003). Procesamiento Digital de Imágenes. Fundamentos y Practicas con Matlab (Digital image processing. Fundamentals and practices with Matlab). En Libro (p. 0). Universidad de León.

2. Beethoven, H. (2012, mayo). Fraude Intelectual, presencia global. http://fce.unal.edu.co/ebooks/fraude/

3. Bensalem, I., Rosso, P., \& Chikhi, S. (2014). Intrinsic Plagiarism Detection using N-gram Classes. EMNLP. https://doi.org/10.3115/v1/D14-1153

4. Carvajal-Rodríguez, A. (2015). Incorporación de la programación informática en el currículum de Biología. Magister, 27(2), 76-82. https://doi.org/10.1016/j.magis.2015.12.002

5. Copyleaks. (2020). Plagriarim Checker para revistas de investigación y documentos Scientefic en línea. Copyleaks. https://copyleaks.com/es/education/research-journals

6. Monjelat, N., \& Rodríguez, G. (2018). Repensando la programación como formación práctica en Ingeniería: Un estudio de caso en primer año. Ingeniare. Revista chilena de ingeniería, 26(1), 172-183. https://doi.org/10.4067/S0718-33052018000100172

7. Moreno, J. M. (2018). Plagio en universidades: Estudio de Turnitin y Compilatio.

8. Septet Systems. (2020). Detector de plagio comprobador de plagio libre. https://www.plagium.com/es/detectordeplagio

9. Siddiqui, N. (2018). Administración de datos en R. The Programming Historian en español. https://doi.org/10.46430/phes0031

10. Sourceforge. (2020). PSeInt es una herramienta para asistir a un estudiante en sus primeros pasos en programación. http://pseint.sourceforge.net/

11. Stamatatos, E. (2009). Intrinsic plagiarism detection using character n-gram profiles. threshold, 2(1,500). 

